\title{
Photometric Observations of the Sun
}

\author{
By GARY A. CHAPMA N \\ San Fernando Observatory, Department of Physics and Astronomy, \\ California State University, Northridge, CA 91330, USA
}

Ground-based calorimetry and photometry of the Sun have been carried out for many years. Following the early years, ground-based photometry has largely replaced ground-based calorimetry, in part due to the advent of airborne and spaceborne detector systems for the broad-band measurement of the solar irradiance and the realization of the difficulty of correcting calorimetry measurements for the effects of the terrestrial atmosphere. Even from spacecraft, recent measurements of the total solar irradiance range from about 1367 to $1374 \mathrm{~W} / \mathrm{m}^{2}$. Most of this difference can be ascribed to differences in instrumental scales, while a variation of about 1 to $2 \mathrm{~W} / \mathrm{m}^{2}$ appears to be due to solar variability. The quiet Sun may also change, globally, over longer time scales. Using disparate data to understand solar variability will require cooperation between a number of current groups, supported by various governments, covering several zones of longitude.

\section{Introduction}

Studying the Sun's output of energy (luminosity) has been a long-standing endeavor. Within the past decade or so, satellites have measured the solar output and have very clearly revealed variability. However, while the satellite measurements have agreed on the actual solar luminosity, agreeing for the most part on the short- and long-term temporal changes. Ground-based measurements, on the other hand, are hampered by the uncertainties in the opacity of the terrestrial atmosphere and its variations and cannot accurately determine the solar luminosity but might be able to measure relative changes in it. This review will attempt to place ground-based efforts into context with space-based experiments. An interesting, broad review of ground-based photometric measurements is that of LaBonte (1988). A general review of solar luminosity variability can be found in (Hudson 1988), Fröhlich et al. (1991) and Fröhlich (1992). We will not review nonimaged programs here. For a good review of these see White (1988) and Livingston (1992).

One of the reasons for carrying out ground-based programs of relative photometry is to help understand the cause of the solar variations seen by satellites. Most of the satellite data have been obtained without any spatial resolution on the solar disk. By having photometric images obtained at selected wavelengths, the satellite measurements can be modeled using selected contrast features seen in ground-based images. We assume that there is a non-changing quiet Sun output and that photometrically detectable features add to or subtract from this quiet Sun output. For relative ground-based photometry, the (assumed) quiet Sun is our photometric standard. If this assumption is not true, we can only find out by doing our very best in removing the effects of features on the solar output.

It has been shown that most of the variations can be attributed to magnetic features (Foukal \& Lean 1988; Chapman et al. 1992). By modeling their effects on the solar output, we hope to gain a better understanding of the flow of energy in and around a solar active region and of the solar dynamo itself. A few questions still unanswered are: (1) where does the thermal energy go that is diverted by sunspots, (2) how much of this energy, if any, is stored in the convection zone, (3) what fraction of a sunspot's missing energy is radiated by faculae and network and over what time scale? 


\section{Past and recent work}

\subsection{The effect of sunspots on solar irradiance}

Attempts in the 1980's to understand the cause of variations in total irradiance measurements relied on synoptic observations of sunspot area and positions. An early example is that of Willson et al. (1981) in which about half of the variance could be removed from the ACRIM I signal (Hudson 1988, and references therein). The sunspot effect was modeled by a Photometric Sunspot Index (PSI) in which it was assumed that the temperature structure of all sunspots is the same and that their contrast does not depend on position on the disk. The quiet Sun limb darkening was assumed to be that of the Eddington gray atmosphere. A similar index was derived in Foukal (1981) (see also Hudson et al. 1982). Because of its widespread use we give the PSI $(\Psi)$ :

$$
P S I=\alpha \sum_{i} A_{s}^{i} \mu_{i}\left(3 \mu_{i}+2\right) / 2
$$

where the sum is taken over all sunspots, $A_{s}$ is the corrected sunspot area in microhemispheres and $\mu_{i}$ is the cosine of the heliocentric angle for the $i$ 'th sunspot. The value of $\alpha$ was found to be 0.33 , but more about that later. This simple form has no free parameters. On the other hand, it may be overly simple. Although several authors quote a mean umbral to spot area of 0.17 , this ratio can vary over a wide range. A more detailed model is the sunspot blocking of Hoyt \& Eddy (1982) where they no longer assume the Eddington gray limb darkening law and they include the umbral and penumbral areas separately. Donnelly et al. (1992) found for the period 1980-1982 that the PSI and the Hoyt-Eddy sunspot blocking function were correlated with a correlation coefficient of 0.92. However, the accuracy of this source of umbral areas is not known. Lean \& Foukal (1990) found a linear regression between their sunspot blocking, $P_{s}$, (identical to 2.1 ) and the Hoyt-Eddy relation with a correlation coefficient of 0.997 but where the $P_{s}$ values were systematically higher by about $20 \%$. Also, the contrast of individual sunspots can be quite variable (Brandt et al. 1990; Steinegger et al. 1990; Beck \& Chapman 1993). It has been known for some time that individual sunspots vary and that umbrae can have darker or brighter parts.

\subsection{The effect of faculae on solar irradiance}

Bright features are associated with magnetic fields. However, the intensity-magnetic field relationship is more complicated than for sunspots. Common terms are faculae, network (active and quiet), plage, and filigree. Faculae have long been seen in white light beyond a radius on the solar disk of about 0.7 to $0.8(\mu \approx 2 / 3)$. The network, which at high resolution has the appearance of filigree, is seen most readily using narrow-band filters or spectroheliographs tuned into the core or near-wings of absorption lines. We understand that these features are connected to flux tubes or collections of flux tubes and that their appearance is determined partly by their magnetic flux distribution and partly by their position on the solar disk Wilson (1981), Zwaan (1987), and references therein. We will refer to these non-spot magnetic features as faculae or flux tubes for the time being. As with sunspots, a simple model has been developed to describe the irradiance fluctuation due to faculae, called the Photometric Facular Index (PFI) (Foukal 1981; Hirayama et al. 1984). As with the PSI, the quiet Sun limb darkening is assumed gray. We show here the simple expression of Chapman et al. (1992):

$$
P F I=C_{p} \sum_{i} A_{p}^{i} \mu_{i}\left(3 \mu_{i}+2\right)\left(\frac{1}{\mu_{i}}-a\right)
$$


where $A_{p}^{i}$ refers to the corrected area in millionths of the solar hemisphere of an active region plage as seen on spectroheliograms obtained in the central Aor so of the Ca II Kline. The parameters $C_{p}$ and $a$ are empirically determined. The parameter $a$ determines whether the faculae are dark, bright, or invisible at disk center. An early attempt to combine parameters for sunspots and faculae by Sofia et al. (1982) used an assumed ratio of facular to sunspot areas rather than relying on published areas of $\mathrm{Ca}$ II plage.

Plage areas may be subject to significant measurement uncertainties. When the McMath-Hulburt Observatory closed, the Ca II plage observations, which were used to determine the published Ca II plage area, were obtained from Sacramento Peak Observatory, Mt. Wilson Observatory, and then Big Bear Solar Observatory. During this time interval there was a change in the plage brightness index which was anticorrelated with plage area. The plage brightness increased by about $50 \%$ during this time interval (Schatten et al. 1985).

An example of the use of sunspot and plage data in modeling solar irradiance variations over long time periods is shown by Foukal \& Lean (1988). The sunspot irradiance deficits were derived from a sunspot "blocking" function virtually the same as 2.1 . The facular irradiance excess was derived from a model that used full disk images obtained in the $\mathrm{HeI} 1083 \mathrm{~nm}$ line. HeI may be a more objective measure of magnetic activity than Ca II plage areas, which were often estimated by eye.

In Chapman \& Meyer (1986), $C_{p}$ was found to be 0.019 when $a$ was assumed to be 0.0 . However, Vrs̃nak et al. (1991) found that $C_{p}$ correlates with the plage intensity index $(r>0.67)$ and has a mean value of about $0.0166 \pm 0.01$. This is fairly close to the value of 0.0185 mentioned in Chapman (1987) and probably well within the limits of accuracy for plage areas themselves.

One problem with using plage areas to define a facular index is that the $\mathrm{Ca}$ II plage covers a larger area than the faculae because of fringing of the magnetic field lines in the chromosphere and more extensive heating. Another problem is that the network elements that lie outside the boundaries determined by the $\mathrm{Ca}$ II plage are not included. If we are attempting to model satellite measurements in the UV we may need a three-component model (Lean et al. 1982). Pap et al. (1992) studied the variation in the ACRIM I signal, smoothed with a 27-day running mean and fitted with a two-component model. The irradiance deficits were based on sunspot blocking and the excesses were based on the Ca II plages. There was a significant discrepancy in 1980 and at other times suggesting that two parameters may be insufficient for a good fit to total irradiance data.

Ground-based, full-disk photometry can model the variations in total irradiance from satellites fairly well. For example, in a recent study (Chapman et al. 1992) fluctuations in the ACRIM I data were fit to about $100 \mathrm{ppm}$ over a 21-day interval using sunspot and facular terms based on 2.1 and 2.2. In this study, the quantity $A_{p}$ represented elements of faculae rather than plage. The facular elements were defined by a $10 \AA$ bandpass filter in the center of the Ca II K-line as detected by the Cartesian Full Disk Telescope (CFDT1) at the SFO, using a pixel size of $5^{\prime \prime} \times 5^{\prime \prime}$. Each detected facular pixel had a contrast at disk center of $4.8 \%$ increasing with $1 / \mu$ (in order to compensate for selection effects caused by the increasing contrast towards the limb). The values of $C_{p}$ and $a$ from this study were found to be 0.030 and 1.1 , respectively, where $a=1.1$ means that the faculae are dark at disk center, having a contrast of $-0.93 \%$. (This value is not inconsistent with values mentioned by Topka et al. (1992) using very high spatial resolution images.) Sunspots were determined from pixels having a contrast of $-8.5 \%$ in photometric images, 


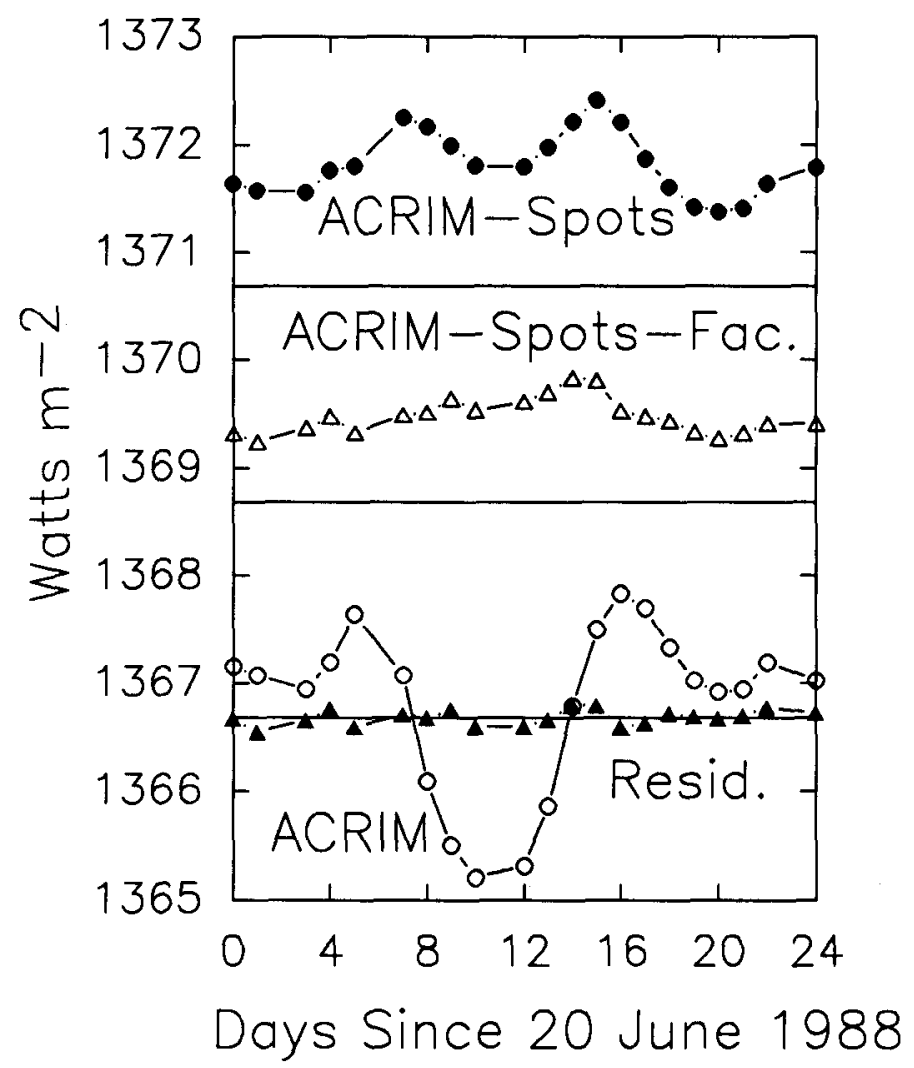

Figure 1. Plot of the ACRIM I signal, ACRIM I minus modeled spot contribution, ACRIM I minus both modeled spot and facular contributions, and residuals from fitting spot, facular, and NOAA-9 (SBUV/2) contributions to ACRIM I. The ACRIM I minus spot and faculae has been shifted upward by $2 \mathrm{~W} / \mathrm{m}^{2}$, and the ACRIM I minus spots by $4 \mathrm{~W} / \mathrm{m}^{2}$, for clarity (from Chapman et al. 1992).

(6723 $\AA$ ). A photometric deficit (DEF) was calculated according to

$$
D E F=\sum_{i} \frac{\Delta I_{i}}{I} \phi\left(\mu_{i}\right)
$$

where $\Delta I_{i} / I$ is the the contrast of a pixel, and $\phi$ is the quiet Sun limb darkening at that same value of $\mu$. During this brief interval in 1988 from 20 June to 14 July, for 21 days the ACRIM I total irradiance could be fit to the sunspot deficit and a modeled facular excess with a coefficient of multiple determination, $R^{2}=0.974$ (see Figure 1). For 109 days of data from June through December in 1988, the total irradiance from Nimbus-7 was fit to sunspot deficits and the same facular excess model as above. The value of $R^{2}$ was 0.9018 , with a standard deviation of approximately $140 \mathrm{ppm}$ (Chapman et al. 1994a). Use of the Mg II core-wing ratio from NOAA-9 (Donnelly 1992) to account for faint network elements, improved the correlations in both of these studies by about 0.01 in the value of $R^{2}$.

Other full-disk photometric measurements have been recently reported by Nishikawa (1990a, 1990b), Nishikawa \& Hirayama (1990), and Lin \& Kuhn (1992). Nishikawa 
(1990b) finds that the active region network has a contribution to fluctuations of the total irradiance that is approximately half that of active region faculae.

Other photometric observations of the full solar disk, restricted to the limb regions, can also be fit to variations in satellite measurements of the total irradiance. Kroll et al. (1990) and Kroll \& Hill (1991) used the Santa Catalina Laboratory for Experimental Relativity by Astrometry (SCLERA) telescope to fit changes in limb darkening, associated with solar activity, to variations in the solar irradiance. Kuhn \& Libbrecht (1985) and Kuhn et al. (1988) have fit measurements of latitudinal fluctuations in photospheric temperature near the solar limb, obtained from the Princeton Distortion Telescope (PDT), to variations in the ACRIM I total irradiance. Kuhn et al. (1988) have been able to obtain fits to total irradiance measurements from satellites with high correlations. Kroll et al. (1990), on the other hand, have found evidence for a change in the limb darkening that is not like the solar cycle. Observations of just the limb may make the effects of active regions across the solar disk difficult to identify due to the time lag for regions to travel from central parts of the solar disk to the limbs.

The contrast of flux tubes and faculae as a function of center-to-limb distance is poorly known. Even the sign of the contrast with $\mu$ is in some dispute (Chapman \& Klabunde 1982; Libbrecht \& Kuhn 1984, 1985; Wang \& Zirin 1987; Lawrence \& Chapman 1988; Foukal et al. 1993; Chapman \& Zielger 1993). Figure 2 shows several different measurements of the center-to-limb variation (CLV) in the contrast of faculae in the mid-visible. The different amplitudes can be explained partly by the differing spatial resolution of the observations. However, part of the discrepancy may have to do with different definitions of contrast. Clearly, the CLV of faculae needs more observational work, preferably photometric, because of the ability to digitize data at high speed and sum them in real time in order to overcome the low signal to noise ratio and the large intensity gradient at the limb. We must be careful to remember that whatever the contrast of flux tubes is in the visible, their contrasts in the UV and IR are quite different (Bonnet et al. 1982; Foukal et al. 1990, 1993).

For modeling total solar irradiance changes, a central question concerns the bolometric contrast of faculae. The contrast of flux tubes in the mid-visible may systematically vary according to magnetic flux (cf. Stenflo \& Harvey 1985). There is evidence that smaller magnetic elements have a positive contrast near disk center, whereas larger magnetic elements have zero or negative contrast near disk center (Lawrence et al. 1991, 1993; Topka et al. 1992). Some of these features are reported to have contrasts of about $30 \%$ when viewed under good seeing and when corrected for losses in contrast due to diffraction effects (Muller \& Keil 1983; Solanki \& Brigljević 1992).

These features would all have strong positive contrasts if viewed with a spectroheliograph (Lawrence et al. 1991), in an absorption line with a narrow filter (Topka et al. 1992), or in the $1600 \AA$ region corresponding to the temperature minimum (Bonnet et al 1982). What is needed is the bolometric contrast as a function of disk position and magnetic flux. For example, the UV spectral irradiance of non-spot, magnetic flux elements can be important, perhaps contributing as much as one-fifth to one-third of the long-term variations in the total irradiance variation (Lean 1989; London et al. 1989).

\section{Recent work}

\subsection{Sunspots}

Several recent studies have shown that the average contrast of sunspots is not constant (Brandt et al. 1990; Steinegger et al. 1990; Beck \& Chapman 1993; Chapman et al. 


\section{Summary of CLV of faculae}

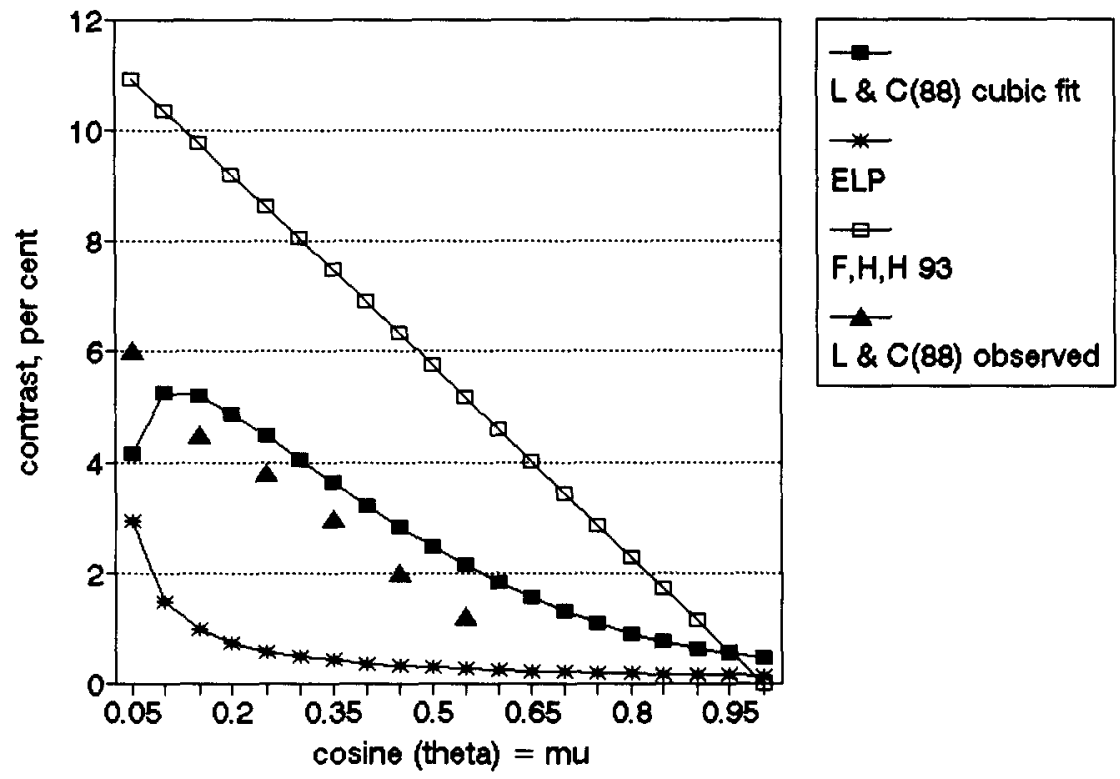

FIgURE 2. Facular contrast from several sources. The filled squares are from a cubic fit to S-series diode array observations (Lawrence \& Chapman 1988), the asterisks are from ELP observations (Chapman \& Ziegler 1993), the open squares are from observations at the NSO (Foukal et al. 1993), and the filled pyramids are based on S-series diode array observations (Lawrence \& Chapman 1988).

1994b). First, there is evidence that the value of $\alpha$ used in 2.1 is too large; the mean value should be near 0.29 rather than 0.33 or 0.35 . There are two points involved here. First, the observed contrast of a sunspot must be properly corrected for stray light. Second, the mean bolometric contrast will determine the correct contribution to the fluctuation in the total irradiance. For these purposes, it is not particularly useful to measure the darkest part of the umbra as has often been done in the past. Furthermore, without imaging the sunspot it is difficult to know much about the distribution of intensity within the umbra. What is needed is a properly weighted, bolometric, mean sunspot contrast.

From an inspection of 2.1 , we can see that $\alpha$ is the mean bolometric contrast of a sunspot, which can be determined from the effective temperature of the entire sunspot, using the Stefan-Boltzmann law. Recent high resolution measurements of sunspots have been made by Brandt et al. (1990) and Steinegger et al. (1990). They showed a wide variation in $\alpha$ between different sunspot groups, from about 0.15 to approximately 0.4 . Furthermore, they showed that $\alpha$ is correlated with the area of the sunspot group. For 130 spots and spot groups, they found that $\alpha=0.2231+0.0244 * \log \left(A_{s}\right)$. The mean for all groups was about 0.27 (Brandt et al. 1992). Beck \& Chapman (1993) found that $\alpha=0.220 \pm 0.016+(0.340 \pm 0.058) * A_{u} / A_{p}$ with a correlation coefficient of 0.602 $(p<0.005)$, where $A_{u}$ and $A_{p}$ are the umbral and penumbral areas, respectively. The mean value of $\alpha$ for 63 sunspot groups was 0.304 .

Another important question concerning $\alpha$ is whether or not it is independent of position 


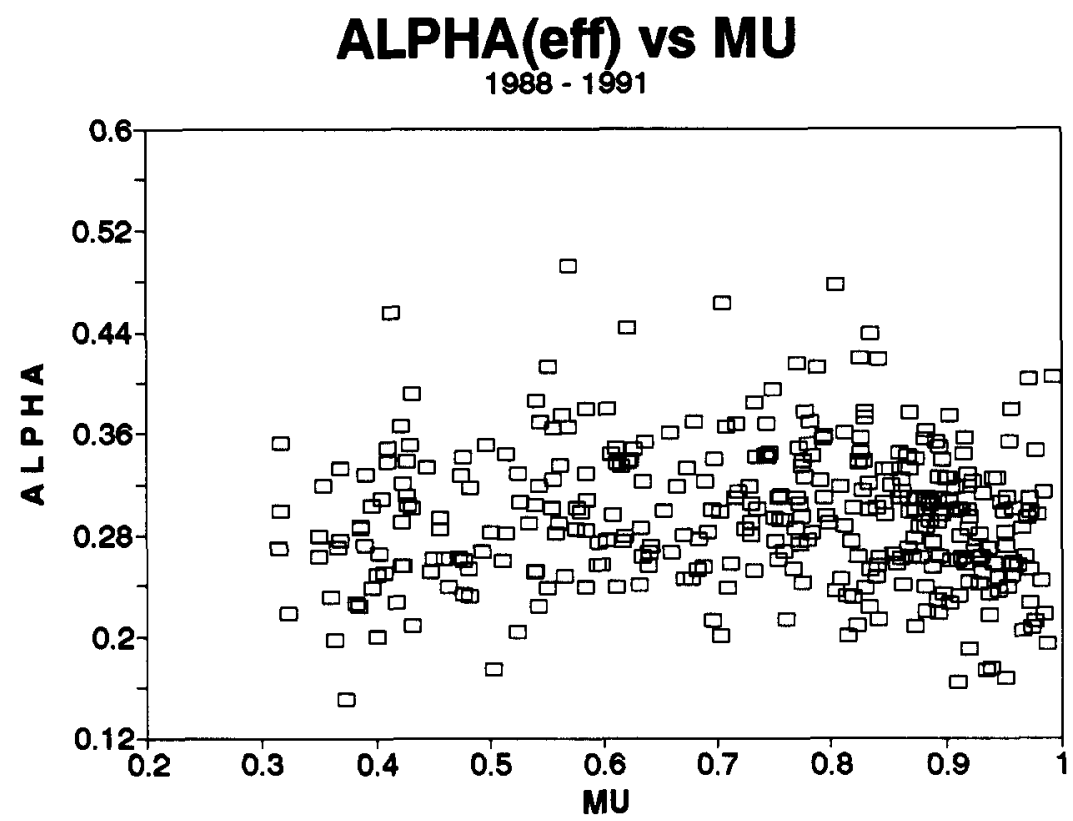

Figure 3. Bolometric contrast, $\alpha_{\text {eff }}$, versus $\mu$ for 377 observations of sunspot groups from 1988 through 1992 (from Chapman et al. 1994b).

on the disk. From a study of 377 measurements of sunspot groups at SFO, during their disk transit, an effective $\alpha\left(\alpha_{e f f}\right)$ has been determined (Chapman et al. 1994b). The value of $\alpha_{e f f}$ is given by

$$
\alpha_{e f f}=D E F / 2 \Psi
$$

where $D E F(2.3)$ is the photometrically measured sunspot deficit and $\Psi$ is given by 2.1 with the sunspot areas being determined from the same pixels from the same photometric full disk images as for the quantity $D E F$. The wavelength of these images is $6732 \AA$. They found that $\alpha_{\text {eff }}=0.218 \pm 0.018+(0.683 \pm 0.028) A_{u} / A_{s}$ with $r^{2}=0.878(N=86)$. The mean value of $\alpha_{\text {eff }}$ for these measurements is 0.293 , very close to values of $\alpha$ from other studies, mentioned above. The value of $\alpha_{\text {eff }}$ appears to be relatively free from the effects of stray light; its variation shows no correlation with sky transparency measured at $530 \mathrm{~nm}\left(r^{2}=0.00065\right)$. Finally, Chapman et al. (1994b) found no dependence of $\alpha_{e f f}$ on $\mu$, the cosine of the heliocentric angle $(r=0.0005 ; N=377)$. Figure 3 shows the quantity $\alpha_{e f f}$ plotted as a function of $\mu$.

\subsection{Non-sunspot magnetic features}

\subsubsection{Brightness related to magnetic flux}

As we are finding, non-spot magnetic features such as faculae or photospheric network can be bright or dark, depending on their location on the disk, their wavelength, and their magnetic flux. All non-spot magnetic features are bright when seen at a wavelength corresponding to the upper photosphere. The wings of the Ca II $\mathrm{K}$ line are formed there and are very useful in mapping out the location of non-spot magnetic features. However, knowing the bolometric contrast requires knowing something about the magnetic flux in each feature. This in turn requires high spatial resolution, and a magnetogram signal that is linear with magnetic flux. For example, if two magnetic features of equal flux but 
opposite polarity are not spatially resolved their flux will cancel in the magnetogram but their positive contrast in the wing of the $\mathrm{K}$ line will not cancel.

An interesting way of modeling irradiance variations is to convert the non-spot magnetic signal in a magnetogram into an irradiance fluctuation. To the extent that irradiance variations are caused by magnetic features (and there is yet no convincing evidence that this is not the case) we want to detect only magnetic features. Furthermore, since a magnetogram is a differential signal, the limb darkening is removed. Such an analysis was carried out with Mt. Wilson magnetograms (Chapman \& Boyden 1986) and should be carried out with KPNO magnetograms. Harvey (1992) has used the total magnetic flux from KPNO magnetograms as an indicator of the level of solar activity in comparison to other solar measurements. Data from the newly installed Spectro-Magnetograph (Jones et al. 1992) should be even more useful for irradiance studies because it will have full disk information on both the continuum and the line core.

Detailed analyses on the behavior of flux tubes in the continuum as a function of magnetic flux and disk position have been carried out by a group using Lockheed's Solar Optical Universal Polarimeter (SOUP) in the Canary Islands (Topka et al. 1992) and a group using the Video Spectra-Spectroheliograph (VSSHG) at the San Fernando Observatory (Lawrence et al. 1991, 1993).

Title et al. (1992), from filtergrams having a spatial resolution approaching $0.35^{\prime \prime}$, find that all bright continuum features have a magnetic signal, but that the reverse is not true, some magnetic features do not have associated bright points either in the continuum or in the line center of Ni I, at $6768 \AA$. Topka et al. (1992), from more extensive data using SOUP for four active regions at various locations on the solar disk, found that the continuum contrast for all magnetic fluxes was negative at $\theta=5^{\circ}$, but became zero at $20^{\circ}$ for a magnetic signal of about 400 Gauss.

Lawrence et al. (1991) using data from the VSSHG, show much the same results, but due to the lower spatial resolution the contrasts of the flux tubes are lower and the magnetic flux where the maximum positive continuum contrasts are seen, away from disk center, are 180 Gauss compared to about 400 Gauss for the SOUP data. Lawrence et al. (1991) calculated the irradiance effects of collections of magnetic flux tubes, based on their contrast and their limb position, taking into account the weighting of the quiet Sun. Depending on the method of defining the quiet Sun, they found an irradiance variation of from $0.3 \pm 0.1 \mathrm{ppm}$ to about $2.1 \pm 0.7 \mathrm{ppm}$ near disk center $(0.79<\mu<0.97)$. If the zero contrast is defined by the line core contrast being less than $4 \%$ then the average quiet Sun background is shifted an average of $(-0.06 \pm 0.02) \%$ relative to defining the zero contrast by those pixels having a magnetic flux less than 80 Gauss. For low and high spatial resolution, determining the quiet Sun intensity is a difficult problem.

\subsubsection{Synoptic measurements of bright features}

While it is desirable to have very high resolution magnetic and brightness maps of the Sun at several wavelengths, this not yet generally done. We do have the daily NSO/KPNO spectra-magnetograph program at $6302.5 \AA$ with $1^{\prime \prime}$ pixels. However, the core of 6302.5 does not sample as high in the solar atmosphere as the wings of the Ca II $\mathrm{K}$-line. On the other hand, the several synoptic programs that obtain full disk Ca II $\mathrm{K}$-line images do not obtain full-disk magnetograms with a possible exception being the NSO/KPNO. Presently, the NSO/SPO Ca II K-line images are obtained photographically with very high spectral resolution. Of particular interest are images obtained in the wing of the K-line, between 1 and $3 \AA$ from line center. At this spectral position, the complicating effects of the chromosphere are mostly avoided. At San Fernando Observatory (SFO), K-line images are obtained with filters having a $10 \AA$ and $3 \AA$ bandpass centered 
on the K-line. The effective passband is split into two passbands on either side of the center of the K-line in the region 1 to $4 \AA$ from line center. Other filter-defined monitors are at Big Bear Solar Observatory (BBSO), the National Solar Observatory's Kitt Peak National Observatory (NSO/KPNO), and Mees Solar Observatory (MSO). These last three use two-dimensional CCD detectors, whereas at SFO linear diode arrays are used. At BBSO the filter has a bandpass of $1.2 \AA$ and a pixel size of $4^{\prime \prime}$ (Marquette 1992). The NSO/KPNO filter has a bandpass of $1 \mathrm{~nm}$ and a pixel size of about 2.4" (Harvey 1994). The MSO has two K-line systems with one designed for daily full disk images. It has a bandpass of $0.3 \AA$ and a pixel size of $5^{\prime \prime} \times 5^{\prime \prime}$ (Labonte, private communication). At SFO, beginning in July 1992, another photometric telescope (CFDT2) with a pixel size of $2.5^{\prime \prime} \mathrm{X} 2.5^{\prime \prime}$ went into operation. The new telescope, in addition to other filters, has a $10 \AA$ and a $3 \AA$ filter at the $\mathrm{K}$-line. The Freiburg group is beginning a study of faculae as well as carrying on with work on sunspots. Details of their, and other, photometric programs are lacking at this time. Apparently a new program of full disk photometry at several wavelengths is being started at BBSO.

A simple global quantity is the irradiance excess of bright features obtained by adding up the contrast of each bright pixel weighted by the quiet Sun limb darkening. Changes in this quantity from day to day can then be compared with changes in spectral irradiance from satellite measurements. For 125 days during the interval from 3 October 1991 to 1 April 1992, the SFO $10 \AA \mathrm{K}$-line spectral irradiance excess data ( $5^{\prime \prime}$ pixels) are highly correlated with the ratio of the SOLSTICE core-to-wing intensity of the Mg II $\mathrm{h}$ and $\mathrm{k}$ line $\left(R_{M g I I}\right.$, White, private communication). In a linear regression (Wiener 1994 ) the following relation was found; for the SOLSTICE core-to-wing ratio $R_{M g I I}=$ $0.1537 \pm 0.0021+(3.98 \pm 0.16) \times 10^{-6}$ times the CFDT1 $10 \AA \mathrm{K}$-line irradiance in ppm relative to the quiet Sun $\left(r^{2}=0.842\right)$. The facular area from this instrument, for 27 January 1991, interpolated using data from 25 to 29 January 1991, was 44,000 microhemispheres, which was 17,000 micro-hemispheres larger (about 60\%) than the area determined by BBSO from their Ca II plage data for the same date (Marquette 1992). Considering the different criteria for a "plage element", the agreement is not bad.

\section{Future work}

\subsection{Improvements in spatial and temporal resolution}

With improved temporal coverage of the solar irradiance by spacecraft, it will become more important to increase the tempo of ground-based measurements by having more stations around the world. Improvements in temporal resolution are needed for those times when significant changes in the solar irradiance occur over a short time span. How much of this change is due to sunspots growing or shrinking, how much is due to "bright" magnetic features growing or shrinking, and how much is due to the changing aspect of these features? An examination of the ingress of two sunspot groups around 27 June 1988 show a change of about $1 \mathrm{~W} / \mathrm{m}^{2}$ (about $730 \mathrm{ppm}$ ) per day in the ACRIM I irradiance. Such a rapid change implies that in order to model total irradiance data from a precision satellite experiment with a precision of order $100 \mathrm{ppm}$, ground-based observations should be taken with intervals of not more than a few hours. This will require irradiance monitors at a number of longitudes, similar to those of the GONG.

The spatial resolution needed to better understand satellite measurements, or to independently determine solar irradiance variations, is not clear. At SFO, measurements of the facular irradiance excess in the K-line with a $10 \AA$ bandpass filter are in very close agreement whether the pixel size is $5^{\prime \prime}$ or $2.5^{\prime \prime}$. On the other hand, the area and irradi- 
ance fluctuations seen with the $3 \AA$ filter and $2.5^{\prime \prime}$ pixels are about twice those obtained with the $10 \AA$ filter.

Presumably, with smaller pixel size and active stabilization the contrast with a $10 \AA$ filter will be higher and the detected number of bright pixels will also go up. A narrower bandpass should help too. These are things to be considered in the SunRISE program, particularly the Precision Solar Photometric Telescopes (PSPT's) undergoing design at the NSO (Foukal et al. 1990; Kuhn 1994).

\section{Summary}

This is an exciting time for ground-based photometric observations of the Sun. It is important, over the next decade, to sort out solar from terrestrial effects on possible climate changes. Yet there are fundamental problems in understanding the cause of solar variability. Their solution will require, among other things, the acquisition of high precision photometric images across the visible spectrum. The solution to these problems will require high quality data and careful data analysis. The most useful data will be from full disk photometric instruments and instruments with polarimetric capabilities, in order to. sort out the influence of magnetic flux. Progress is being made, in spite of great difficulties. In closing, it is argued that ground-based photometry, using photoeletric detector systems, can make a significant contribution to our understanding of variations in the solar output. This is a challenging task for the solar community.

Acknowledgments. I appreciate direct and indirect help in preparing this review, and in some of the research presented here, from the following: A. M. Cookson, J. J. Dobias, J. Lawrence, C. Mach, and S. R. Walton, and financial support from NASA Grant NAGW-3017 and NSF Grant ATM-9115111.

\section{REFERENCES}

Brandt, P. N., Schmidt, W. \& Steinegger, M. 1990 On the area ratio of sunspots. Solar Phys. (Lett.) 129, 191-194.

Brandt, P. N., Schmidt, W. \& Steinegger, M. 1992 Photometry of sunspots observed at Tenerife. In Proceedings of the Workshop on the Solar Electromagnetic Radiation Study for Solar Cycle 22 (ed. R. F. Donnelly). pp. 130-141. NOAA ERL SEL, Boulder, CO, USA.

Beck, J. G. \& Chapman, G. A. 1993 A study of the contrast of sunspots from photometric images. Solar Phys. 146, 49-60.

Bonnet, R. M., Bruner, M., Acton, L. W., Brown, W. A., Decaudin, M. \& Foing, B. 1982 Rocket photographs of fine structure and wave patterns in the solar temperature minimum. Astron. Astrophys. 111, 125-129.

BRUNING, D. H. \& LABONTE, B. J. 1983 Interpretation of solar irradiance variations using ground-based observations. Ap. J. 271, 853-858.

Chapman, G. A. \& Klabunde, D. P. 1982 Measurements of the limb darkening of faculae near the solar limb. Ap. J. 261, 387-395.

Chapman, G. A., Herzog, A. D. \& Lawrence, J. K. 1986 Time-integrated energy budget of a solar activity complex. Nature 319, 654-655.

Chapman, G. A. \& Boyden J. E. 1986 Solar irradiance variations derived from magnetograms. Ap. J. 302, L71-L73.

Chapman, G. A. \& MeYer, A. D. 1986 Solar irradiance variations from photometry of active regions. Solar Phys. 103, 21-31.

Chapman, G. A. 1987 Variations of solar irradiance due to magnetic activity. Ann. Revs. Astron. Astrophys. 25, 633-667. 
Chapman, G. A., Herzog, A. D., Lawrence, J. K., Walton, S. R., Hudson, H. S. \& FISHER, B. M. 1992 Precise ground-based solar photometry and variations of total irradiance. J. Geophys. Res. 97, 8,211-8,219.

Chapman, G. A., Cookson, A. M. \& Hoyt, D. V. 1994a Solar irradiance from Nimbus-7 compared with ground-based photometry. Solar Phys., in press.

Chapman, G. A., Cookson, A. M. \& Dobias, J. J. 1994b Observations of changes in the bolometric contrast of sunspots. Ap. J., submitted.

Chapman, G. A. \& Ziegler, B. 1993 Analysis of 1983 observations with an extreme limb photometer. Solar Phys., submitted.

Donnelly, R. F., Hudson, H. S., PAP, J. \& Willson, R. C. 1992 Estimating solar chromospheric UV fluxes from sunspot and solar radio data. In Proceedings of the Workshop on Electromagnetic Radiation Study for Solar Cycle 22 (ed. R.F. Donnelly). pp. 275-285. NOAA ERL SEL, Boulder, CO, USA.

Donnelly, R. F., ED. 1992 Proceedings of the Workshop on Electromagnetic Radiation Study for Solar Cycle 22 NOAA ERL SEL, Boulder, CO, USA.

FoukAL, P. V. 1981. Sunspots and changes in the global output of the Sun. In The Physics of Sunspots (ed. L.E. Cram \& J.E. Thomas). pp. 391-423. Sacramento Peak Observatory, Sunspot, NM, USA.

FOUKAL. P. \& LEAN, J. 1986 The influence of faculae on total solar irradiance and luminosity. Ap. J. 302, 826-835.

FoUKAL, P. \& LEAN, J. 1988 Magnetic modulation of solar luminosity by photospheric activity. Ap. J. 328, 347-357.

Foukal, P. V., Brueckner, G. E., Hudson, H. S., Livingston, W. C., Noyes, R. W. \& RoBle, R. G. 1990 RISE, Radiative Inputs of the Sun to Earth: A Research Plan for the 1990s on Solar Irradiance Variations. Prepared for The Division of Atmospheric Sciences, National Science Foundation.

Foukal, P., Little, R., Graves, J., Rabin, D. \& Lynch, D. 1990 Infrared imaging of faculae at the deepest photospheric layers. Ap. J. 353, 712-715.

Foukal, P., Harvey, K. \& Hill, F. 1993 Do changes in the photospheric magnetic network cause the 11-year variation of total solar irradiance? Ap. J. (Lett.), submitted.

FröHLICH, C. \& PAP, J. 1989 Multi-spectral analysis of total solar irradiance variations. Astron. Astrophys. 220, 272-280.

Fröhlich, C., Foukal, P. V., Hickey, J. R., Hudson, H. S. \& Willson, R. C. 1991 Solar irradiance variability from modern measurements. In The Sun in Time (ed. C.P. Sonett, M.S. Giampapa \& M.S. Matthews). pp. 11-29. Univ. Arizona Press, Tucson, AZ, USA.

FröHLICH, C. 1992 Solar irradiance variability. In Proceedings of the Workshop on Electromagnetic Radiation Study for Solar Cycle 22 (ed R.F. Donnelley). pp. 1-10. NOAA ERL SEL, Boulder, CO, USA.

HARVEY, K. L. 1992 Measurements of solar magnetic fields as an indicator of solar activity evolution. In Proceedings of the Workshop on the Solar Electromagnetic Radiation Study for Solar Cycle 22 (ed. R. F. Donnelly). pp. 113-129. NOAA ERL SEL, Boulder, CO, USA.

HARVEY, K. 1994 Irradiance models based on solar magnetic fields. In The Sun as a Variable Star: Solar and Stellar Irradiance Variations (ed. J.M. Pap, C. Fröhlich, H.S. Hudson \& S.K. Solanki). Cambridge Univ. Press, in press.

Hirayama. T., OKamoto, T. \& Hudson, H. S. 1984 Facular limb darkening functions for irradiance modeling. In Solar Irradiance Variations on Active Region Time Scales (ed. B. J. LaBonte, G. A. Chapman, H. S. Hudson \& R. C. Willson). NASA CP-2310, 59-71.

Hoyt, D. V. \& EdDY, J. A. 1982 An Atlas of Variations in the Solar Constant caused by Sunspot Blocking and Facular Emissions from 1874 to 1981. NCAR Tech. Note TN-194, Boulder, CO, USA.

Hudson, H. S. 1988 Observed variability of the solar luminosity. Ann. Revs. Astron. Astrophys. 26, 473-507.

Hudson, H. S. Silva, S., Woodard, M. \& Willson, R. C. 1982 The effects of sunspots on 
solar irradiance. Solar Phys. 76, 211-219.

Jones, H. P., Duvall, JR., T. L., Harvey, J. W., Mahaffey, C. T., Schwitters, J. D. \& Simmons, J. E. 1992 The NASA/NSO spectromagnetograph. Solar Phys. 139, 211-232.

Kroll, R. J., Hill, H. A. \& BeARdsley, B. J. 1990 A correlation between changes in solar luminosity and differential radius measurements. In Climate Impact of Solar Variability (ed. K. H. Schatten \& A. Arking). NASA CP-3086, pp. 65-72.

Kroll, R. J. \& HILL, H. A. 1991 The SCLERA program for monitoring solar limb darkening and diameter. Poster paper presented at the NSF/NCAR Workshop on Solar-Terrestrial Impacts on Global Change, Boulder, CO, USA, May 8-10, 1991.

Kuhn, J. R., LibBrecht, K. G. \& Dicke, R. H. 1988 The surface temperature of the Sun and changes in the solar constant. Science 242, 908-911.

KUHN, J. R. \& LiBbRECHT, K. G. 1985 Observations of a solar latitude dependent limb brightness variation. $A p . J .290,758-764$.

Kunn, J. R 1994 Brightness observations of the Sun. In The Sun as a Variable Star: Solar and Stellar Irradiance Observations (ed. J.M. Pap, C. Fröhlich, H.S. Hudson \& S.K. Solanki). Cambridge Univ. Press, in press.

LABONTE, B. J. 1988 Ground-based photometric measurements. In Solar Radiative Output Variations (ed. P. V. Foukal). pp. 156-175. NCAR, Boulder, CO, USA.

LAWRENCE, J. K. \& Chapman, G. A. 1988 Photometric observations of facular contrasts near the solar limb. Ap. J. 335, 996-1004.

Lawrence, J. K., Chapman, G. A. \& Walton, S. R. 1991 Weak magnetic fields and solar irradiance variations. $A p$. $J$. 375, 771-774.

LaWrence, J. K., Topka, K. P. \& Jones, H. P. 1993 Contrast of faculae near the disk center and solar variability. em J. Geophys. Res. 98, 18,911-18,918.

LEAN, J. 1988 Empirical modeling of UV and EUV flux variations. In Solar Radiative Output Variations (ed. P. Foukal). pp. 113-124. NCAR, Boulder, CO, USA.

LEAN, J. \& FOUKAL, P. 1988 A model of solar luminosity modulation by magnetic activity between 1954 and 1984. Science 240, 906-908.

LEAN, J. \& FoukAL, P. 1990 Solar cycle modulation of total irradiance: an empicrical model from 1874 to 1988 . In Climate Impact of Solar Variability (ed. K. H. Schatten \& A. Arking). NASA-3086, pp. 197-204.

Lean, J. L., White, O. R., Livingston, W. C., Heath, D. F., Donnelly, R. F. \& SkuMANICH, A. 1982 A three-component model of the variability of the solar ultraviolet flux. J. Geophys. Res. 87, 10,307-10,317.

LEAN, J. 1989 Contribution of ultraviolet irradiance variations to changes in the Sun's total irradiance. Science 244, 197-200.

Libbrecht, K. G. \& KuHN, J. R. 1984 A new measurement of the facular contrast near the solar limb. Ap. J. 277, 889-896.

LibBrecht, K. G. \& KunN, J. R. 1985 On the facular contrast near the solar limb. Ap. J. 299, 1047-1050.

LIN, H. \& KuHN, J.R. 1992 Precision IR and visible solar photometry, I. Solar Phys. 141, 1-26.

Livingston, W. C. 1992 Observations of solar spectral irradiance variations at visible wavelengths. In Proceedings of the Workshop on Solar Electromagnetic Radiation Study for Solar Cycle 22 (ed. R.F. Donnelly). pp. 11-19. NOAA ERL SEL, Boulder, CO, USA.

London, J., J. PAP \& G.J. RotTMAn 1989 Observed solar near UV variability: A contribution to variation of the solar constant. In Handbook for Middle Atmosphere Program (ed. J. Lastovićka, T. Miles \& A. O'Neill). Vol. 29, pp. 9.

Marquette, W. H. 1992 In Proceedings of the Workshop on Solar Electromagnetic Radiation Study for Solar Cycle 22 (ed. R.F. Donnelly). pp. 154-159. NOAA ERL SEL, Boulder, CO, USA.

MUller, R. A. \& KeIL, S. L. 1983 The characteristic size and brightness of facular points in the quiet photosphere. Solar Phys. 87, 243-250. 
NIsHIKAWA, J. 1990 A new system of precise full-disk solar surface photometry. Ap. J. Suppl. 74, 315-323.

NisHIKAWA, J. 1990 Estimation of total irradiance variations with the CCD solar surface photometer. Ap. J. 359, 235-245.

Nishikawa, J. \& HiRayama, T. 1990 Facular structures derived from precise two-color contrast observations. Solar Phys. 127, 211-228.

PAP, J., Willson, R.C. \& DonNelly, R.F. 1992 Two-parameter model of total solar irradiance variation over the solar cycle. In The Solar Cycle (ed. K. Harvey). ASP 27, 491-502.

Schatten, K. H., Miller, N., Sofia, S., Endal, A. S., Chapman, G., \& Hickey, J. 1985 The importance of improved facular observations in understanding solar constant variations. Ap. J. 294, 689-696.

Solanki, S. K. \& Brigljević, V. 1992 Continuum brightness of solar magnetic elements. Astron. Astrophys. 262, L29-L32.

SofiA, S., Oster, L. \& SChatTen, K. 1982 Solar irradiance modulations by active regions during 1980. Solar Phys. 80, 87-98.

Steinegger, M., Brandt, P. N., PaP, J. \& Schmidt, W. 1990 Sunspot photometry and the total solar irradiance deficit measured in 1980 by ACRIM. Astrophysics. Space Sci. 170, 127-133.

Stenflo, J., O. \& HaRVey, J. W. 1985 Dependence of the properties of magnetic fluxtubes on area factor or amount of flux. Solar Phys. 95, 99-118.

Title, A. M., Topka, K. P., Tarbell, T. D., Schmidt, W., Balke, C. \& Scharmer, G. 1992 On the differences between plage and quiet Sun in the solar photosphere. Ap. J. 393, 782-794.

Topka, K. P., Tarbell, T. D. \& Title, A. M. 1992 Properties of the smallest solar magnetic elements I. Facular contrast near Sun center. Ap. J. 396, 351-363.

VRs̃nak. B., PlačKo, D. \& RUždJaK, V. 1991 Calcium plage intensity and solar irradiance variations. Solar Phys. 133, 205-213.

WANG, H. \& ZIRIN, H. 1987 The contrast of faculae near the solar limb. Solar Phys. 110, 281-293.

WhIte, O. R. 1988 Ground-based surrogates for UV and EUV fluxes. In Solar Radiative Output Variations (ed. P. Foukal). pp. 88-112. NCAR, Boulder, CO, 1988.

WIENER, L. 1994 M. S. thesis, CSU, Northridge (in progress).

Wilson, P. R. 1981 Faculae, filigree, and calcium bright points. Solar Phys. 69, 9-14.

Willson, R. C., Gulkis, S., Janssen, M., Hudson, H. S. \& Chapman, G. A. 1981 Observations of solar irradiance variability. Science 211, 700-702.

ZWAAN, C. 1987 Elements and patterns in the solar magnetic field. Ann. Revs. Astron. Astrophys 25, 83-111. 